\title{
GALAXY SED FITTING FROM AKARI TO HERSCHEL: $0.7<z<4$ SUB-MILLIMETER LYMAN BREAK GALAXIES IN INFRARED
}

\author{
D. Burgarella and the PEP-HerMES-COSMOS teAm \\ Laboratoire d'Astrophysique de Marseille, Technopole de Chateau-Gombert, 13013 Marseille, France \\ Aix-Marseille University \\ E-mail: denis.burgarella@oamp.fr \\ (Received July 01, 2012; Accepted August 15, 2012)
}

\begin{abstract}
Lyman break Galaxies are galaxies selected in the rest-frame ultraviolet. But, one important and missing information for these Lyman break galaxies is the amount of dust attenuation. This is crucial to estimate the total star formation rate of this class of objects and, ultimately, the cosmic star formation density. AKARI, Spitzer and Herschel are therefore the major facilities that could provide us with this information. As part of the Herschel Multi-tiered Extragalactic Survey, we have began investigating the rest-frame far-infrared properties of a sample of more than 4,800 Lyman Break Galaxies in the GOODS-North fiels. Most LBGs are not detected individually, but we do detect a sub-sample of 12 objects at $0.7<z<1.6$ and one object at $z=2.0$. The ones detected by Herschel SPIRE have redder observed NUV-U and U-R colors than the others, while the undetected ones have colors consistent with average LBGs at $z>2.5$. We have analysed their UV-to-FIR spectral energy distributions using the code CIGALE to estimate their physical parameters. We find that LBGs detected by SPIRE are high mass, luminous infrared galaxies. They also appear to be located in a triangle-shaped region in the $A_{\mathrm{FUV}}$ vs. $\log L_{\mathrm{FUV}}$ diagram limited by $A_{\mathrm{FUV}}=0$ at the bottom and by a diagonal following the temporal evolution of the most massive galaxies from the bottom-right to the top-left of the diagram. In a second step, we move to the larger COSMOS field where we have been able to detect 80 Lyman break galaxies (out of $\sim 15,600$ ) in the far infrared. They form the largest sample of Lyman break galaxies at $\mathrm{z}$ $>2.5$ detected in the far-infrared. We tentatively name them Submillimeter Lyman break galaxies (S-LBGs).
\end{abstract}

Key words: infrared: telescope; conferences: proceedings

\section{INTRODUCTION}

Dropout galaxies are selected from a color-color diagram that highlights the presence of the Lyman break in the far-ultraviolet (FUV). This Lyman break technique selects star-forming galaxies at all redshifts. Depending on the redshift that one desires to explore, a different set of filters is used to detect the Lyman break feature.

The safest way to estimate the total star formation rates (SFR) of galaxies is to consider the energy budget involving far-ultraviolet (FUV) and far-infrared (FIR) measurements (e.g., Buat, 1996). But, because only a small number of individual Lyman Break Galaxies (LBGs) have been detected in the FIR/submm range (Chapman et al., 2000; Chapman et al., 2009; Siana et al., 2009) we need to observe this type of galaxies at lower redshifts to understand their dust emission. Burgarella et al. (2007) detected dropout galaxies at $z \sim 1$ at $24 \mu \mathrm{m}$ with Spitzer. But, the dust luminosities estimated from the rest frame $12 \mu \mathrm{m}$ flux density is far from the peak of the dust emission and could provide 

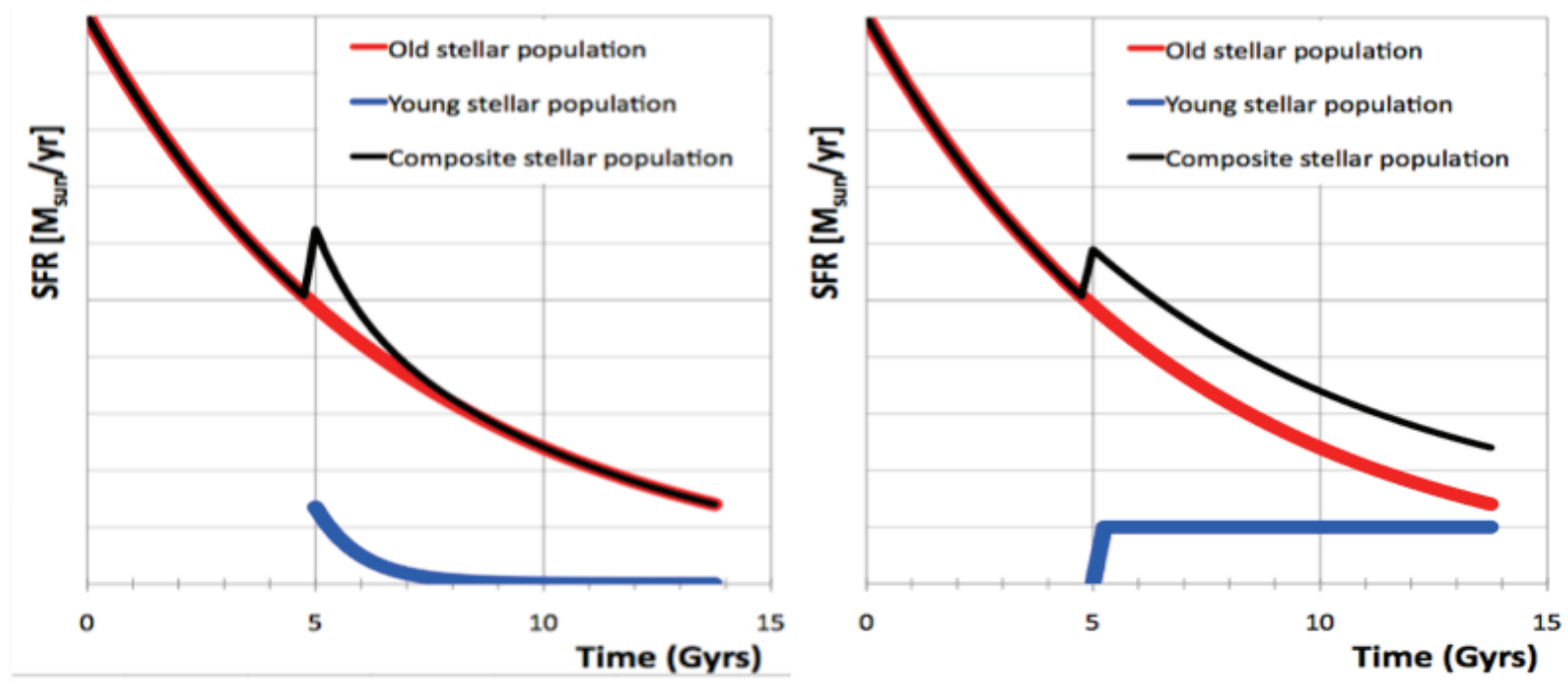

Fig. 1. The various star formation histories that could be used by CIGALE for two stellar populations.

biased SFR estimates.

It is therefore useful and most interesting to move to the far-infrared where we better sample the dust emission of the galaxies.

\section{OBSERVATIONS}

We observe in the FIR a sample of LBGs at $0.7<z<1.6$ (FUV dropouts), at $1.6<z<2.8$ (nearUV or NUV dropouts) and finally at $2.5<z<4.0$. We use observations from HerMES (Oliver et al., 2010) ${ }^{1}$. This is the first opportunity to directly estimate the dust luminosity (or upper limits) of unlensed LBGs from FIR data. We assume $\Omega_{\mathrm{m}}=0.3, \Omega_{\Lambda}=0.7$, and $H_{0}=70 \mathrm{~km} \mathrm{~s}^{-1} \mathrm{Mpc}^{-1}$ and use $\mathrm{AB}$ magnitudes throughout.

We also used AKARI, an infrared telescope launched by ISAS/JAXA in 2006 (Murakami et al., 2007), that successfully carried out an all sky survey at mid- and far-infrared wavelengths, as well as conducting several large area surveys and obtaining many other pointed observations across the wavelength range $2-160 \mu \mathrm{m}$. One of these deep pointings on the Chandra Deep Field South with the IRC, an instrument designed to carry out near- to mid-infrared imaging allowed us to constrain the Spectral Energy Distribution (SED) of the LBGs in the rest-frame mid-infrared.

The photometry is performed with IRAF DAOPHOT II (Stetson, 1987) in the NUV, and in the FUV with the NUV coordinates. We use CIGALE (Noll et al., 2009) to build models in the range $0 \leq z \leq 3$ and delimit the regions corresponding to LBGs at $0.7<z<1.6,1.6<z<2.8$ and at $2.5<z<4.0$ in the color-color diagrams ${ }^{2}$.

\footnotetext{
${ }^{2}$ Note that the code accounts for IGM attenuation.
}

\footnotetext{
${ }^{1}$ http://hermes.sussex.ac.uk.
} 

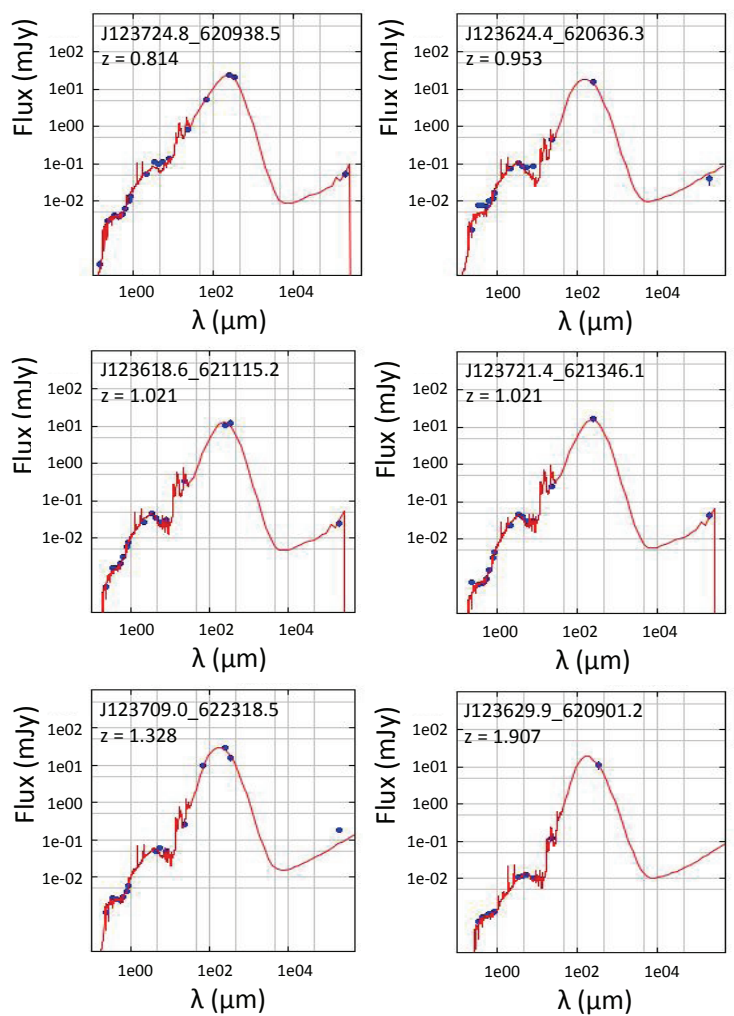

Fig. 2. Observed spectral energy distributions of LBGs superimposed on best fit models $\left(S_{\nu}[\mathrm{mJy}]\right.$ vs. $\left.\log \lambda[\mu \mathrm{m}]\right)$.

\section{WHAT IS CIGALE?}

CIGALE is a code developed by our team at LAM. It is available for download ${ }^{3}$. It performs a SED fitting from the far-ultraviolet to the millimeter range using either the best-fit, a Bayesian-like or a Monte-Carlo Markov Chain analysis. Dust luminosities $\left(L_{\mathrm{IR}}=\right.$ $L[8-1,000 \mu \mathrm{m}])$ and other parameters are estimated using CIGALE (Noll et al., 2009). One can select among two single stellar population libraries (Fig. 1) and several IR models/templates. An AGN component can also be added to estimate the AGN fraction (contribution by a potential AGN to $L_{\mathrm{IR}}$ ). It is important to stress that the parameters of the dust attenuation law can be modified and CIGALE allows for two separate stellar populations with a multiphase dust treatment.

\footnotetext{
${ }^{3}$ http://www.oamp.fr/cigale.
}

\section{RESULTS}

For the lowest redshift samples, individual SEDs with the best models selected by CIGALE are shown for the five LBGs with radio data and for the $(z=1.9)$ LBG. Fig. 2 shows that CIGALE is able to fit the SEDs from the FUV to the radio successfully. The average FUV luminosity of the SPIRE detected objects is $\log \left\langle\left(L_{\mathrm{FUV}} / \mathrm{L}_{\odot}\right)\right\rangle=(10.7 \pm 0.2)$, and most of them are therefore UV-Luminous Galaxies. Their average dust luminosity is $\log \left\langle\left(L_{\mathrm{IR}} / \mathrm{L}_{\odot}\right)\right\rangle=(11.9 \pm 0.1)$ and their stellar average mass is $\log \left\langle\left(M_{*} / \mathrm{M}_{\odot}\right)\right\rangle=(11.0 \pm 0.5)$. A comparison with Magdis et al. (2010) shows that the average stellar mass is similar to the average stellar mass $\left(\log \left\langle\left(M_{*} / \mathrm{M}_{\odot}\right)\right\rangle=11\right)$ of $z \sim 3$ LBGs detected at $\lambda=8 \mu \mathrm{m}$.

However, the main result is that the vast majority of the dropout galaxies are not detected by SPIRE with the present detection limits.

Reddy et al. (2010) and Bouwens et al. (2010) suggest that objects with a lower UV luminosity at $1.5 \leq z \leq 2.6$ have lower bolometric luminosities than UV-bright galaxies which, in turn, may suggest lower dust attenuations if we account for the relation between bolometric luminosity and dust attenuation. However, when computing $\mathrm{A}_{F U V}$ from the stacked points of Reddy et al. (2010), we find a trend similar to ours for most of the points. Carilli et al. (2008) and Ho et al. (2008) estimate the UV dust attenuation by comparing radio-based star formation rates to UV-based ones using a stacking analysis. We show in Fig. 3, the two points corresponding to different $\log L_{\mathrm{FUV}}$ and they agree with the above trend. Burgarella et al. (2006) reached a similar conclusion using Spitzer $24 \mu \mathrm{m}$ observations of a sample of LBG at $z \sim 1$. Even though the detected LBGs are not consistent with a trend suggesting a decrease of the dust attenuation with the ultraviolet luminosity, we must acknowledge that most of them are not detected with Herschel. To go beyond these limits, we have divided our $z \sim 1$ sample in two subsamples as a function of $\mathrm{L}_{F U V}$ and stacked them in the $250 \mu \mathrm{m}$ image (Fig. 3). We find the same trend, again. Note, however, that accounting for the error bars, our stacking and Reddy et al.,'s are both consistent with a constant $A_{\mathrm{FUV}}$.

The bottomline of our analysis is that the dust attenuation of LBGs does not decrease but instead might flatten or even slightly increase with decreasing UV luminosities (Fig. 4). UV-faint galaxies might be either 


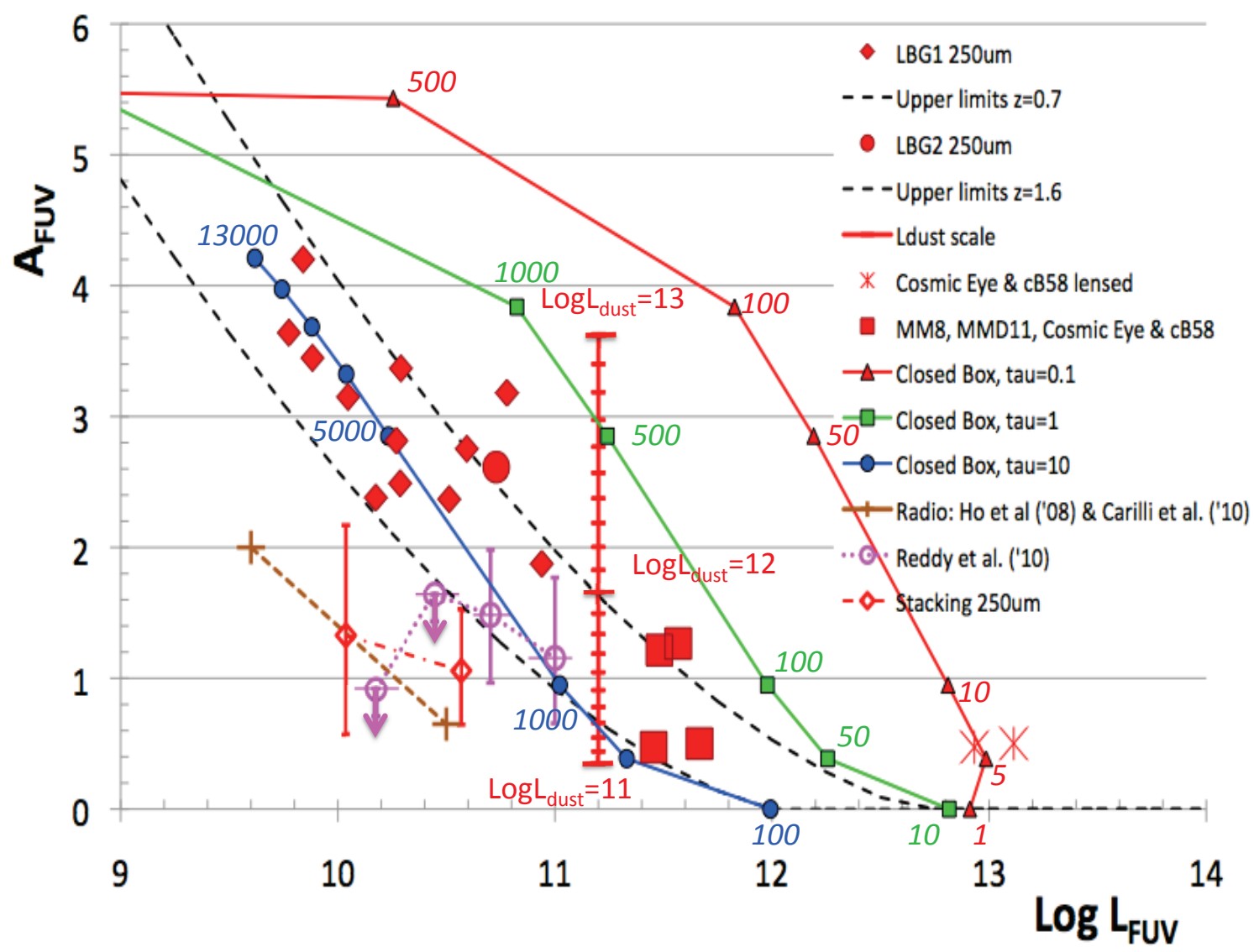

Fig. 3. Red diamonds are LBGs detected by Herschel. The two big red stars are the two high redshift LBGs (the cosmic Eye and cB58) as observed, while the big red squares are the same sources after correcting for the amplification plus two unlensed ones (Siana et al., 2008, 2009; Chapman et al., 2000; Chapman \& Casey, 2009). The blue dots, green boxes and red triangles are the closed-box models, plotted as a function of time (age in Myrs) increasing from the bottom-right to the top-left part of the diagram. All the models would scale to the left with decreasing mass. The vertical scale provides the values of $L_{\mathrm{IR}}$ in steps of $0.1 \mathrm{dex}$. The two crosses linked by a dashed line are radio-based measurements. The purple open dots are the stacked points from Reddy et al. (2010). The red open diamonds correspond to stacked points at $z \sim 1$ from our sample. The bottom line of this figure is that the LBGs appear to be located in a triangle-shaped region in the $A_{\mathrm{FUV}}$ vs. $\log L_{\mathrm{FUV}}=0$ diagram limited by $A_{\mathrm{FUV}}=0$ at the bottom and by a diagonal following the temporal evolution of the most massive galaxies from the bottom-right to the top-left of the diagram. By stacking these Lyman break galaxies, we conclude that the average dust attenuation of UV-faint galaxies does not decrease with luminosity as suggested by other works. The limits of this region are well explained using a closed-box model, where the chemical evolution of galaxies produces metals, which in turn lead to higher dust attenuation when the galaxies age.

galaxies with a low dust attenuation that are very faint and undetected in far-infrared or more massive galaxies with most of their luminosity emitted in the far-infrared but a small part in the ultraviolet. In the latter case, we can envision several options: a shell of dust-free stars surrounding the galaxies, two separate components with one of them providing the far-infrared lumi- nosity and the other one the ultraviolet luminosity or even a dusty galaxy with an inhomogeneous dust covering factor allowing ultraviolet radiation to partially get out of the galaxies with a low dust attenuation. 


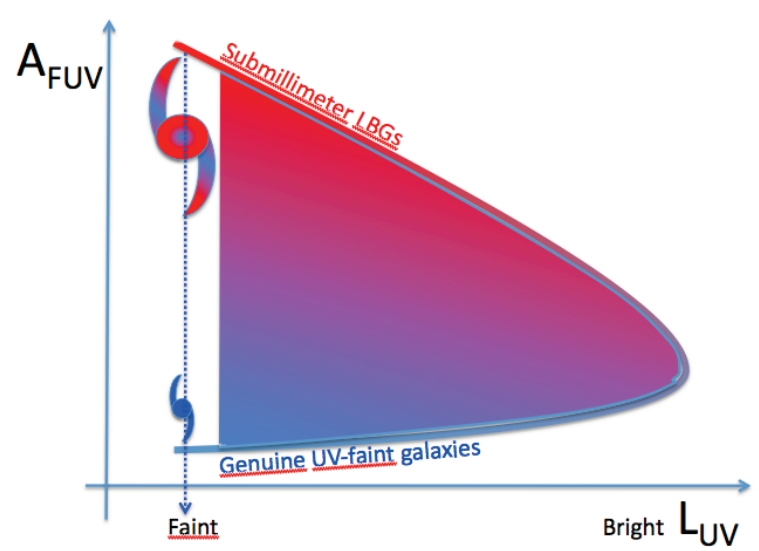

Fig. 4. LBGs can fall in this triangle-shaped area. So, an ultraviolet-faint galaxie could be either genuinely faint in ultraviolet or the ultraviolet emission could be only a small contribution to a much larger bolometric luminosity, most of it being in far-infrared.

\section{A NEW SAMPLE OF SUB-MILLIMETER LY- MAN BREAK GALAXIES AT $2.5<z<4.0$ DE- TECTED IN THE COSMOS FIELD BY HER- SCHEL}

We defined a selection of LBGs in the 2 sq. deg. COSMOS field and from the original sample of about 40,000 objects, about 15,000 do have 30-band photometric redshifts in the range $2.5<\mathrm{z}<4.0$ which form our highredshift LBG sample. 80 of these galaxies are detected in far-infrared with Herschel-SPIRE (Fig. 5) and they form, by far, the largest sample or sub-millimeter LBGs (S-LBGs). These LBGs are tentatively described as a link between more normal Lyman break galaxies and sub-millimeter galaxies. A paper describing the sample and its characteristics is in preparation (Burgarella et al., 2012).

The ultraviolet slope $\beta$ is estimated from the 30 intermediate bands included in the COSMOS database independently of CIGALE. It is interesting to notice from Fig. 6 that the $\beta$ value is different from the three samples defined as i) not detected in infrared, ii) detected up to $24 \mu \mathrm{m}$ and iii) detected up to the farinfrared. This means that the galaxies detected in farinfrared have redder UV slopes than the one undetected and confirms that our detected FIR S-LBGs can indeed be associated to their UV counterparts, at least statistically.

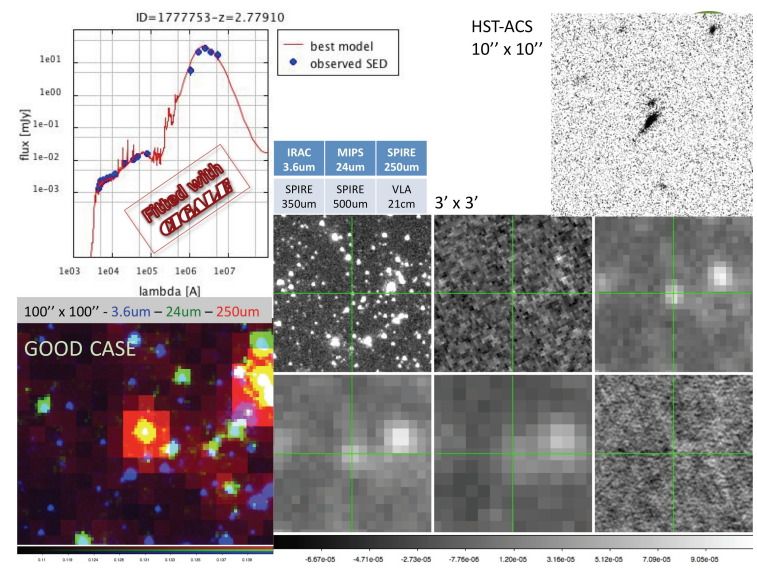

Fig. 5. One of the candidates LBGs detected by Herschel/SPIRE with the associated SED fit by CIGALE.

The Second AKARI Conference was supported by BK21 program to Seoul National University by the Ministry of Education, Science and Technology, Center for Evolution and Origin of Universe (CEOU) at Seoul National University, National Research Foundation Grant No. 2006-341-C00018 to HMLee, Astronomy Program, Seoul National University Nagoya University Global COE Program: Quest for Fundamental Principles in the Universe, Division of Particle and Astrophysical Science, Nagoya University, and Institute of Space and Astronautical Science, Japan Aerospace Exploration Agency. We also thank the Centre National d'Etudes Spatiales for its financial support for the AKARI project.

\section{REFERENCES}

Buat, V. \& Xu, K., 1996, Star Formation and Dust Extinction in Disk Galaxies. Comparison between the UV Non-Ionizing and the FIR Emissions, A\& A, 306, 61

Burgarella, D., Buat, V., \& Iglesias-Páramo, J., 2005, Star Formation and Dust Attenuation Properties in Galaxies from a Statistical Ultraviolet-to-FarInfrared Analysis, MNRAS, 360, 1413

Burgarella, D., Le Floc'h, E., \& Takeuchi, T. T., 2007, Lyman Break Galaxies at $z \sim 1$ and the Evolution of Dust Attenuation in Star-Forming Galaxies with Redshift, MNRAS, 380, 986

Burgarella, D., et al., 2006, Ultraviolet-to-Far Infrared Properties of Lyman Break Galaxies and Luminous Infrared Galaxies at $z \sim 1, \mathrm{~A} \& \mathrm{~A}, 450,69$ 


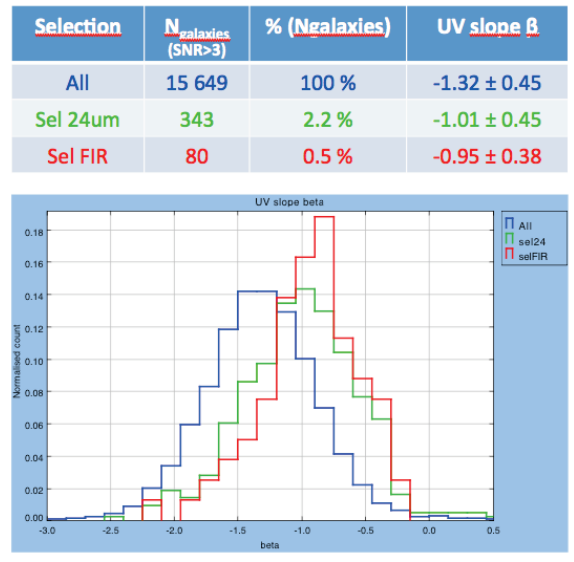

Fig. 6. The colors of the three LBG subsamples (namely undetected in infrared, detected up to $24 \mu \mathrm{m}$ and detected in far-infrared) present clearly different UV slope $\beta$ values. This strongly suggests that the association of the UV object with the far-infrared ones are not pure chance associations.

Burgarella, D., Buat, V., Takeuchi, T. T., Wada, T., \& Pearson, C., 2009, Deep $15 \mu \mathrm{m}$ AKARI Observations in the CDFS: Estimating Dust Luminosities for a MIR-Selected Sample and for Lyman Break Galaxies and the Evolution of $\mathrm{L}_{d u s t} / \mathrm{L}_{U V}$ with the Redshift, PASJ, 61, 177

Burgarella, D., Heinis, S., Magdis, G., et al., 2011, HerMES: Lyman Break Galaxies Individually Detected at $0.7<\mathrm{z}<2.0$ in GOODS-N with Herschel/SPIRE, ApJ, 734, L12

Bouwens, R. J., et al., 2010, Very Blue UV-Continuum Slope ? of Low Luminosity $z \sim 7$ Galaxies from WFC3/IR: Evidence for Extremely Low Metallicities?, ApJ, 708, L69

Carilli, C. L., et al., 2008, Star Formation Rates in Lyman Break Galaxies: Radio Stacking of LBGs in the COSMOS Field and the Sub-?Jy Radio Source Population, AJ, 689, 883

Chapman, S. C., Scott, D., Steidel, C. C., Borys, C., Halpern, M., Morris, S. L., Adelberger, K. L., Dickinson, M., Giavalisco, M., \& Pettini, M., 2000, A Search for the Submillimetre Counterparts to Lyman Break Galaxies, MNRAS, 319, 318

Chapman, S. C. \& Casey, C. M., 2009, Submillimetre Detection of the $\mathrm{z}=2.83$ Lyman-Break Galaxy, Westphal-MM8, and Implications for SCUBA2, MNRAS, 398, 1615

Dale, D. A. \& Helou, G., 2002, The Infrared Spectral Energy Distribution of Normal Star-forming
Galaxies: Calibration at Far-Infrared and Submillimeter Wavelengths, ApJ, 576, 159

Dunlop, J. S., McLure, R. J., Robertson, B. E., Ellis, R. S., Stark, D. P., Cirasuolo, M., \& de Ravel, L., 2012, A Critical Analysis of the Ultraviolet Continuum Slopes $(\beta)$ of High-Redshift Galaxies: No Evidence (Yet) for Extreme Stellar Populations at $z>6$, MNRAS, 420, 901

Ho, I. T., et al., 2010, Dust Obscuration in Lyman Break Galaxies at $z \sim 4$, ApJ, 722, 1051

Maraston, C., 2005, Evolutionary Population Synthesis: Models, Analysis of the Ingredients and Application to High-Z Galaxies, MNRAS, 362, 799

Magdis, G., et al., 2010, On the Stellar Masses of IRAC Detected Lyman Break Galaxies at $z \sim 3$, ApJ, 714, 1740

Murakami, H., et al., 2007, The Infrared Astronomical Mission AKARI, PASJ, 59, 369

Noll, S., Burgarella, D., Giovannoli, E., Buat, V., Marcillac, D., \& Muñoz-Mateos, J. C., 2009, Analysis of galaxy Spectral Energy Distributions from far-UV to far-IR with CIGALE: Studying a SINGS Test Sample, A\&A, 507, 1793

Oliver, S. J., et al., 2010, Specific Star Formation and the Relation to Stellar Mass from $0<z<2$ as Seen in the Far-Infrared at 70 and $160 \mu \mathrm{m}$, A\& A, 518, L21

Reddy, N., et al., 2010, Dust Obscuration and Metallicity at High Redshift: New Inferences from $\mathrm{UV}, \mathrm{H} \alpha$, and $8 \mu \mathrm{m}$ Observations of $z / \operatorname{sim} 2$ Starforming Galaxies, ApJ, 712, 1070

Siana, B., et al., 2009, Detection of Far-Infrared and Polycyclic Aromatic Hydrocarbon Emission from the cosmic Eye: Probing the Dust and Star Formation of Lyman Break Galaxies, ApJ, 698, 1273

Siana, B., et al., 2008, Spitzer Observations of the $\mathrm{z}$ $=2.73$ Lensed Lyman Break Galaxy: MS 1512cB58, ApJ, 689, 59

Stetson, P., 1987, DAOPHOT - A Computer Program for Crowded-Field Stellar Photometry, PASP, 99, 191 